\title{
Estudo quanti-qualitativo sobre amamentação exclusiva por gestantes de alto risco
}

\author{
A quantitative and qualitative study of exclusive breastfeeding \\ intention by high-risk pregnant women
}

Suzely Adas Saliba Moimaz (https://orcid.org/0000-0002-4949-529X) ${ }^{1}$

Denise de Toledo Rós (https://orcid.org/0000-0003-4910-9886) ${ }^{1}$

Tania Adas Saliba (https://orcid.org/0000-0003-1327-2913) ${ }^{1}$

Nemre Adas Saliba (https://orcid.org/0000-0001-9608-1631) ${ }^{1}$

${ }^{1}$ Departamento de Odontologia Preventiva e Social, Faculdade de Odontologia de Araçatuba, Universidade Estadual Paulista Júlio de Mesquita Filho. R. José Bonifácio 1193, Vila Mendonça. 16015-050 Araçatuba SP Brasil.suzely.moimaz@ unesp.br

\begin{abstract}
The scope of this research was to assess the breastfeeding intention among high-risk pregnant women and related factors. It is a cross-sectional, qualitative and quantitative study, with 1,118 high-risk pregnant women who attended the prenatal care unit at a specialized center of the Unified Health System. The dependent variables were exclusive breastfeeding intention, intended duration and conditions that might interfere with breastfeeding. The Epi Info 7.4.1, Bioestat 5.3 and IRAMUTEQ 0.7.2.0 programs were used for data processing. The results showed that $8.76 \%$ of the pregnant women had conditions that could affect lactation. Among the women, $93.83 \%$ affirmed having exclusive breastfeeding intention, of which $69.86 \%$ intended to breastfeed until the child was six months old, revealing an association with sociodemographic variables $(p<0.05)$. The intended breastfeeding duration was related to the age ( $p=0.0041)$, marital status $(p=0.0053)$ and level of education ( $p=0.0116)$. The main reasons reported for not providing exclusive breastfeeding were the following: HIV, use of medications, work and lack of information. This research concluded that a small cohort of high-risk pregnant women presented conditions that could interfere with breastfeeding. Most of them intended to breastfeed exclusively for six months.
\end{abstract}

Key words High-risk pregnancy, Breastfeeding, Qualitative Research
Resumo O objetivo nesta pesquisa foi avaliar a intenção de amamentação de gestantes de alto risco e fatores relacionados. Trata-se de um estudo transversal, quanti-qualitativo, com 1.118 gestantes de alto risco que realizaram pré-natal em centro especializado no Sistema Único de Saúde. As variáveis dependentes foram intenção de amamentação exclusiva, período pretendido e condições que poderiam interferir na amamentação. Para processamento dos dados, foram utilizados o software Epi Info 7.4.1, Bioestat 5.3 e IRAMUTEQ 0.7.2.0. Os resultados mostraram que 8,76\% das gestantes apresentavam condições que poderiam afetar o aleitamento. A pretensão de amamentação exclusiva foi afirmada por 93,83\%, sendo $69,86 \%$ até os seis meses, demonstrando associação com as variáveis sociodemográficas $(p<0,05)$. O período pretendido para amamentar apresentou associação com idade ( $p=0,0041)$, estado civil $(p=0,0053)$ e escolaridade $(p=0,0116)$. Os principais motivos para a recusa da amamentação exclusiva foram os seguintes: HIV, uso de medicação, trabalho e falta de informação. Conclui-se que uma pequena parcela das gestantes de alto risco apresentava condições que poderiam interferir na amamentação. A maioria delas pretendiam amamentar exclusivamente no peito por um período de seis meses.

Palavras-chave Gravidez de alto risco, Aleitamento Materno, Pesquisa Qualitativa 


\section{Introdução}

A amamentação é considerada uma das principais estratégias para a nutrição da criança na primeiríssima infância, acarretando na redução da mortalidade infantil. Por esse motivo, vem sendo amplamente recomendada por instituições internacionais e nacionais, como a Organização Mundial de Saúde (OMS), o Fundo das Nações Unidas para a Infância (UNICEF), a Organização Pan-Americana de Saúde (OPAS), a Aliança Mundial para Ações de Amamentação (World Alliance for Breastfeeding Action - WABA) e o Ministério da Saúde ${ }^{1-5}$.

Para o desenvolvimento e o incentivo de práticas de aleitamento materno por profissionais da área da saúde, a OMS, em parceria com a UNICEF, propôs os Dez Passos para o Sucesso do Aleitamento Materno, em 1992, visando a implantar o Hospital Amigo da Criança ${ }^{6}$. Esses passos, revisados recentemente, abrangem a criação de uma rotina para ser seguida, um treinamento para todos os profissionais de saúde do local, $\mathrm{o}$ incentivo às gestantes sobre os benefícios e a importância da amamentação na primeira hora após o parto, o alojamento conjunto entre mãe e bebê e a desmotivação da inserção de bicos artificiais para crianças em período de amamentação ${ }^{1,7}$.

No Brasil, a estratégia Rede Cegonha - regulamentada pelas Portarias no 1.459 , de 24/06/2011, e $n^{\circ} 650$, de 05/10/2011 - tem a finalidade de estruturar e organizar a atenção à saúde materno-infantil no país por meio de quatro componentes: pré-natal; parto e nascimento; puerpério e atenção integral à saúde da criança; e o sistema logístico ${ }^{8}$.

Os benefícios promovidos pela amamentação são vários tanto para o bebê quanto para a mãe. Dentre os benefícios para o bebê, destacam-se a diminuição de doenças respiratórias e de diarreias, menor desnutrição infantil, melhora no desenvolvimento neurológico, diminuição do risco do desenvolvimento de diabetes e obesidade, contribuição para o desenvolvimento correto do sistema estomatognático e estabelecimento de uma oclusão dentaria favorável. Dentre os benefícios para as mães, sobressaem-se a ajuda na redução do volume uterino e do peso, diminuição do risco de hemorragia pós-parto e diminuição das chances de desenvolvimento de diabetes crônica em casos de diabetes gestacional ${ }^{9-13}$.

O incentivo à amamentação deve ser iniciado ainda na gestação para que possíveis barreiras que possam impedir a amamentação ou acarretar o desmame precoce sejam identificadas e prevenidas assim que possível ${ }^{14}$.
Segundo o Ministério da Saúde, condições físicas, psicológicas ou ambientais da gestante, presentes antes ou durante a gestação, e alterações no desenvolvimento fetal podem levar à denominada gestação de alto risco. Dentre esses fatores, destacam-se os seguintes: alterações no desenvolvimento fetal, gestação gemelar e trigemelar, adolescência, idade avançada, doenças infectocontagiosas e sexualmente transmissíveis, toxoplasmose, tuberculose, lúpus, hipertensão, diabetes, depressão, uso de drogas ilícitas e lícitas, uso de medicamentos contínuos que podem causar danos à gestação ou ao bebê, entre outras ${ }^{15}$.

As gestantes de alto risco merecem atenção especial, tanto nos cuidados durante a gravidez quanto no período de aleitamento, pois algumas condições podem ser desfavoráveis para o aleitamento materno.

Estudos sobre aleitamento materno demonstraram taxas que variaram entre $20 \%$ a $59 \%$ de amamentação exclusiva em crianças menores de seis meses ${ }^{7,13,16-19}$. Para recém-nascidos prematuros de baixo peso, as taxas variam entre $19,5 \%$ a $76 \%$ de aleitamento materno exclusivo no âmbito hospitalar, caindo mais $50 \%$ no período após alta do bebê e o primeiro retorno ao ambulatório $^{19,20}$.

As causas de desmame precoce são diversas, destacando-se a falta de orientação e acompanhamento das mães, a não amamentação na primeira hora após o parto, o parto cesáreo, a condição socioeconômica materna desfavorável, o trabalho materno e a introdução de bicos artificiais e fórmulas lácteas precoces na alimentação

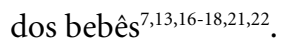

Em relação às gestantes de alto risco, além dos motivos já expostos, podem existir outros fatores impeditivos e/ou complicadores para o aleitamento materno exclusivo, muitas vezes desconhecidos pela maioria delas. As informações sobre as condições em que a amamentação deve ser interrompida ou não deve ser recomendada são pouco divulgadas para as gestantes e suas famílias; além disso, são escassos os estudos com gestantes de alto risco, talvez devido às dificuldades de acesso a elas, sejam nos domicílios ou nos serviços de saúde, referência para atenção secundária.

Considerando a relevância do aleitamento materno para a saúde materno-infantil, o objetivo nesta pesquisa foi verificar a intenção de amamentação por gestantes de alto risco, atendidas no serviço público de saúde. 
Trata-se de uma pesquisa transversal, quantiqualitativa, tipo inquérito, com gestantes de alto risco que realizaram o pré-natal no período de janeiro de 2016 a novembro de 2017 em uma unidade de atenção secundária do SUS.

O Ambulatório Médico de Especialidades (AME) do estado de São Paulo foi selecionado por ser o local de referência do serviço público de saúde para 28 municípios nos casos em que a condição de saúde da mãe e/ou do bebê apresentam alterações que possam comprometer a saúde materno-infantil, ou seja, quando há gestação de alto risco. A partir do AME, as gestantes também podem, em caso de necessidade, ser referidas para a unidade hospitalar terciária, complementando o acompanhamento, no entanto, nesses casos, o vínculo inicial com o AME mantém-se durante toda a gestação.

A amostra foi inicialmente composta por 1.200 gestantes. Entretanto, 82 entrevistas foram perdidas por não responderem aos critérios qualitativos do estudo. Os critérios de inclusão utilizados foram os seguintes: gestantes que apresentavam alterações consideradas de alto risco e que estavam em condições físicas e emocionais para responder à entrevista e gestantes que consentiram em participar da pesquisa.

As entrevistas foram realizadas por pesquisadores calibrados previamente ao estudo, empregando-se formulário específico com questões sobre o perfil das pacientes, motivo para encaminhamento ao serviço especializado, histórico de gestações anteriores, pretensão de amamentação exclusiva e complementar, orientação prévia e apoio familiar para a amamentação. Os motivos para amamentação exclusiva foram investigados por meio de questão aberta. Um estudo piloto foi realizado com 160 gestantes para adequação do formulário e ajustes na coleta de informações; contudo, esses dados não foram utilizados nesta pesquisa.

A abordagem para inclusão das gestantes na pesquisa foi realizada, preferencialmente, na primeira consulta pré-natal de alto risco no local da pesquisa. No entanto, a determinação do risco gestacional ocorre em cada consulta pré-natal de baixo risco realizada nas Unidades de Saúde, visto que alterações na mãe e/ou no feto podem ser identificadas durante todo período gestacional, não existindo período gestacional determinado para o encaminhamento para o pré-natal de alto risco.
As variáveis dependentes do estudo foram a intenção de amamentar de forma exclusiva e a duração pretendida de aleitamento. As variáveis independentes do estudo foram idade, renda familiar, escolaridade, estado civil, ocupação, moradia, apoio familiar para a amamentação, se foi amamentada pela mãe, orientação profissional sobre aleitamento materno, histórico de amamentação, motivo de encaminhamento para o AME e semana gestacional no momento da entrevista. Esta última variável foi incluída considerando que gestantes em período gestacional mais avançado deveriam apresentar maior conhecimento sobre o tema mediante a proximidade do nascimento do bebê.

A questão a respeito da duração pretendida para o aleitamento materno não apresentava opções de respostas pré-estabelecidas. Após o término da pesquisa, para melhor interpretação dos dados, as respostas foram categorizadas da seguinte forma: não pretende amamentar, pretende amamentar por até 1 ano, pretende amamentar por mais de 1 ano e até quando puder/ bebê quiser.

Os antecedentes obstétricos de risco para a gestação atual incluem o histórico de abortos anteriores, cirurgias uterinas, infertilidade, multiparidade e filhos anteriores com alguma restrição de crescimento, prematuridade ou malformações.

Os dados quantitativos foram processados com o auxílio dos softwares Epi Info 7.4.1 (Center for Disease Control and Prevention) e Bioestat 5.3 (Ayres, 2007). Foram realizados o teste qui quadrado e exato de Fisher, quando necessário, para verificar associações entre as variáveis de estudo, aceitando-se um nível de significância de 5\%. Nas análises de associação com o histórico de amamentação, foram excluídas as primigestas, sendo incluídas 701 gestantes. Para as análises sobre a duração pretendida para amamentação foram excluídas as respostas "até quando puder" ou "até quando o bebê quiser" (n = 159), consideradas sem informação.

A seguinte pergunta aberta foi realizada: "Pretende amamentar o seu bebê somente com o leite do seu peito? Se sim, por que pretende? Se não, por que não pretende?”. As respostas foram transcritas na íntegra e, posteriormente, passaram por correção linguística para otimização da análise dos dados por meio da técnica de análise de conteúdo, através da frequência de repetição das palavras, com o uso do software IRAMUTEQ 0.7.2.0 (Interface de R pour lês Analyses Multidimensionnelles de Textes et de Questionnaires). Esse 
software é livre e ancora-se no software R, possibilitando o entendimento de textos e trazendo os resultados apresentados na forma de classificação hierárquica descendente, análise de similitude e nuvem de palavras.

As entrevistadas foram agrupadas em dois segmentos, segundo as respostas obtidas no estudo: gestantes que pretendiam amamentar seus bebês exclusivamente com leite do peito por determinado período e gestantes que não pretendiam amamentar ou iriam fazer a complementação da amamentação logo após o nascimento dos bebês. Para cada grupo foi realizada a análise de similitude que possibilita identificar a conexidade entre as palavras de acordo com sua frequência e com a nuvem de palavras, que gera como resultado uma organização gráfica de acordo com a frequência de cada palavra ${ }^{22}$.

Para todas as gestantes participantes da pesquisa foi solicitada a assinatura do Termo de Consentimento Livre e Esclarecido, que continha explicação detalhada dos objetivos do estudo. Foram respeitados todos os aspectos éticos para pesquisas com seres humanos contidos na Declaração de Helsinki (1964, reformulada em 2008), no Código de Nuremberg e propostos pela Comissão Nacional de Ética em Pesquisa (CONEP - 2012). O estudo também obteve aprovação pelo Comitê de Ética em Pesquisa da UNESP.

\section{Resultados}

As principais condições apresentadas pelas gestantes relacionadas à classificação de gestação de alto risco estão demonstradas na Tabela 1. A pretensão de amamentação exclusiva, o histórico de amamentação, o apoio familiar e a orientação durante a gestação foram descritos na Tabela 2.

A idade das gestantes variou entre 13 e 45 anos, com média de 27,98 (dp $\pm 7,59$ ), sendo a faixa com maior concentração de gestantes a faixa com idades entre 20 a 34 anos $(61,36 \%)$. Houve predomínio de baixa renda $(47,40 \%)$ e escolaridade de até 11 anos (84,08\%). Sobre o tempo da gestação, 48,30\% estavam no segundo trimestre, entre a $13^{\mathrm{a}}$ e $26^{\mathrm{a}}$ semana de gestação. A pretensão de aleitamento materno exclusivo foi associada com gestantes com mais idade ( $\mathrm{p}<0,0001)$, que apresentaram maior renda e escolaridade $(\mathrm{p}=$ 0,0001 e $p=0,0006$, respectivamente), tinham um companheiro $(\mathrm{p}=0,0006)$ e estavam empregadas $(\mathrm{p}=0,0007)$ (Tabela 3$)$.

O maior período de duração pretendida pelas gestantes de alto risco, para amamentação foi associado com maior idade ( $\mathrm{p}=0,0041)$, menor renda $(\mathrm{p}=0,0243)$, maior escolaridade $(\mathrm{p}=$ $0,0116)$, vivem com companheiro $(p=0,0053)$ e sem emprego $(p=0,0420)$ (Tabela 4$)$.

As gestantes que tiveram histórico de amamentação de outros filhos apresentaram associação com maior escolaridade $(p=0,0472)$, apoio familiar $(\mathrm{p}=0,0494)$, pretensão de realizar a amamentação exclusiva $(\mathrm{p}=0,0165)$ e pretensão de amamentar por tempo maior $(\mathrm{p}<0,0001)$.

A análise da questão aberta está demonstrada nas Figuras 1 e 2. Nas análises das respostas do grupo de gestantes que pretendiam amamentar exclusivamente com leite do peito, foram registradas 8099 ocorrências de palavras, sendo 444 formas ativas, com média de repetição de 7,72, em 1049 textos. Foram incluídas palavras com frequência mínima de 7 repetições, para melhor compreensão e clareza da figura. Observando-se a análise de similitude representada e a nuvem de palavras, presente na Figura 1, notou-se que as ocorrências de palavras mais frequentes foram as seguintes: bebê, criança, saúde, saudável e bom.

$\mathrm{Na}$ análise das respostas do grupo de gestantes que não pretendiam realizar o aleitamento exclusivo foram observadas 400 ocorrências de palavras, sendo 148 formas ativas, com média de repetição de 5,8, em 69 textos. A análise de similitude e a nuvem de palavras estão representadas na Figura 2, demonstrando como palavras mais frequentes as seguintes: não, saber, trabalhar, HIV e água.

\section{Discussão}

Neste estudo sobre pretensão de amamentação de gestantes de alto risco, notou-se que algumas gestantes apresentavam condição desfavorável ao aleitamento materno. No entanto, quase a totalidade das gestantes pretendia amamentar exclusivamente com o leite do peito por algum período. A intenção de amamentação exclusiva esteve associada com as variáveis sociodemográficas e o histórico de amamentação anterior. Os principais motivos reportados para a amamentação exclusiva estiveram relacionados à saúde do bebê; já os principais motivos para não realizar o aleitamento exclusivo foram a condição sistêmica da mãe, o trabalho e a falta de informação.

São poucas as contraindicações para a amamentação, dentre quais se destacam as seguintes: mães portadoras do vírus do HIV; uso de medicamentos, como antineoplásicos; e bebês portadores de galactosemia, que não podem consumir 
Tabela 1. Condições e afecções apresentadas pelas gestantes consideradas de alto risco, de 28 municípios do estado de SP, 2018 ( $\mathrm{n}=1.118)$.

\begin{tabular}{llc}
\hline & & $\mathbf{n}(\mathbf{\%})$ \\
\hline Condições que & Vírus da Imunodeficiência Adquirida (HIV) & $9(0,81)$ \\
podem contraindicar & Lúpus & $5(0,45)$ \\
e /ou interromper & Epilepsia & $12(1,07)$ \\
temporariamente o & Transtornos mentais & $32(2,86)$ \\
aleitamento materno & Hanseníase & $1(0,09)$ \\
& Tuberculose & $1(0,09)$ \\
& Neoplasias & $4(0,36)$ \\
& Uso de drogas lícitas e ilícitas & $31(2,77)$ \\
& Varicela & $1(0,09)$ \\
Outras condições frequentes & Herpes & $2(0,18)$ \\
& Idade materna $>$ 20 e $\leq 35$ anos & $434(38,82)$ \\
& Hipertensão & $356(31,84)$ \\
& Diabetes & $120(10,73)$ \\
& Doenças Sexualmente Transmissíveis (DST) & $58(5,19)$ \\
& Antecedentes obstétricos de risco & $69(6,17)$ \\
& Princípio de aborto & $67(5,99)$ \\
& Malformação fetal & $35(3,13)$ \\
& Hepatite & $7(0,63)$ \\
& Ginecopatias & $64(5,72)$ \\
\hline
\end{tabular}

Nota: Diversas entrevistadas apresentaram mais de uma condição de alto risco.

nenhum tipo de lactose. A hepatite $B$, apesar de ser transmitida pelo leite materno, não contraindica a amamentação quando o bebê é imunizado contra a doença ${ }^{4}$. Foram poucas as gestantes de alto risco neste estudo que apresentavam essas condições.

A transmissão vertical do HIV pode ocorrer no período gestacional ou por meio do leite materno. Durante a gestação, o uso do coquetel retroviral é essencial para evitar a transmissão vertical do HIV. Porém, com relação à amamentação, ainda são necessários mais estudos que comprovem a efetividade das medicações, de forma que não é recomendado o aleitamento materno nesse caso $^{23-26}$. Para essas gestantes, o acompanhamento e a orientação durante a gestação e o pós-parto tornam-se fundamentais para minimizar a transmissão viral e amenizar os desconfortos e os problemas mamários decorrentes da não amamentação ${ }^{24,25}$.

A interrupção temporária do aleitamento é recomendada em casos de infecções herpéticas, com vesículas em mama, de varicela poucos dias antes do parto e de doença de Chagas com sangramento mamário ou em fase aguda e abscesso mamário. Em situações de uso de drogas lícitas ou ilícitas, o leite deve ser desprezado por meio de ordenha, no período recomendado para cada situação, podendo-se retornar à amamentação após esse período ${ }^{6}$.

O leite materno é o principal alimento para garantir o crescimento do bebê nos primeiros seis meses de vida ${ }^{16}$. A amamentação também proporciona a proteção da criança contra infecções e o contato entre mãe e filho, criando um vínculo que leva ao sentimento de segurança ao bebêt $\hat{A}^{7,27}$. O melhor desenvolvimento intelectual de crianças amamentadas por mais de seis meses e a diminuição do risco a obesidade infantil também foram reportados na literatura ${ }^{11,28}$.

A OMS recomenda que, nos seis primeiros meses de amamentação, seja oferecido somente o leite do peito ao bebê e, após esse período, a continuação do aleitamento até os dois anos de $v \operatorname{lda}^{6}$. Neste estudo, foi observado que a maioria das gestantes pretendia amamentar até um ano de vida do bebê.

$\mathrm{O}$ apoio às mães no que concerne a amamentação pode ser um fator diferencial para o sucesso do aleitamento materno ${ }^{7,9,29,30}$. Esse apoio pode ser tanto profissional quanto familiar. Alguns estudos que fizeram o acompanhamento de profissionais de saúde com lactantes demonstraram que, com ajuda para superar possíveis barreiras para a amamentação, com grande frequência há resultados positivos ${ }^{29,31}$. O apoio à amamentação 
Tabela 2. Distribuição numérica e percentual segundo a pretensão e o histórico de amamentação por gestantes de alto risco. SP, $2018(\mathrm{n}=1.118)$.

\begin{tabular}{|c|c|c|}
\hline Variáveis & & n (\%) \\
\hline \multirow{2}{*}{$\begin{array}{l}\text { Pretende amamentar exclusivamente } \\
\text { com o leite do peito? }\end{array}$} & Sim & $1.049(93,83)$ \\
\hline & Não & $69(6,17)$ \\
\hline \multirow{4}{*}{$\begin{array}{l}\text { Por quanto tempo pretende amamentar } \\
\text { de forma exclusiva? }\end{array}$} & Não pretende & $69(6,17)$ \\
\hline & Até 4 meses & $209(18,69)$ \\
\hline & Até 6 meses & $781(69,86)$ \\
\hline & Mais de 6 meses & $59(5,28)$ \\
\hline \multirow{6}{*}{$\begin{array}{l}\text { Por quanto tempo pretende } \\
\text { amamentar? }\end{array}$} & Não pretende amamentar & $40(3,57)$ \\
\hline & Até 6 meses & $236(21,11)$ \\
\hline & Até 1 ano & $399(35,69)$ \\
\hline & Até 2 anos & $242(21,65)$ \\
\hline & Mais de 2 anos & $42(3,76)$ \\
\hline & Até quando puder/bebê quiser & $159(14,22)$ \\
\hline \multirow[t]{3}{*}{ Foi amamentada pela sua mãe? } & $\operatorname{Sim}$ & $820(73,35)$ \\
\hline & Não & $215(19,23)$ \\
\hline & Não sabe & $83(7,42)$ \\
\hline \multirow[t]{2}{*}{ Alguém te apoia a amamentar? } & Sim & $834(74,60)$ \\
\hline & Não & $284(25,40)$ \\
\hline \multirow{2}{*}{$\begin{array}{l}\text { Recebeu orientação sobre } \\
\text { amamentação? }\end{array}$} & Sim & $251(22,45)$ \\
\hline & Não & $867(77,55)$ \\
\hline \multirow[t]{5}{*}{ Quantos filhos nascidos vivos? } & Nenhum & $417(37,30)$ \\
\hline & 1 filho & $379(33,90)$ \\
\hline & 2 filhos & $185(16,55)$ \\
\hline & 3 ou mais filhos & $137(12,25)$ \\
\hline & Não tem outros filhos & $417(37,30)$ \\
\hline \multirow[t]{4}{*}{ Seus outros filhos foram amamentados? } & Não amamentou & $88(12,55)$ \\
\hline & 1 filho & $339(48,36)$ \\
\hline & 2 filhos & $165(23,54)$ \\
\hline & 3 ou mais filhos & $109(15,55)$ \\
\hline \multirow[t]{6}{*}{ Por quanto tempo amamentou? } & Não amamentou & $88(12,55)$ \\
\hline & Até 4 meses & $132(18,83)$ \\
\hline & Até 6 meses & $113(16,12)$ \\
\hline & Ate 1 ano & $171(24,40)$ \\
\hline & Ate 2,5 anos & $162(23,11)$ \\
\hline & Mais de 2,5 anos & $35(4,99)$ \\
\hline
\end{tabular}

deve ser iniciado ainda no cuidado pré-natal, principalmente com gestantes primigestas e que não conseguiram amamentar ${ }^{32}$.

A orientação profissional sobre amamentação também é considerada como um ponto importante para o sucesso da amamentação. Na literatura, diversos estudos relatam associações positivas entre orientações educativas e a redução do risco da não amamentação ${ }^{17,29,32,33}$. Nesta pesquisa com gestantes de alto risco, grande parte não tinha recebido nenhuma orientação sobre aleitamento até o momento da entrevista, ficando evidenciada a falta de informação das gestantes que não pretendiam realizar a amamentação exclusiva através das palavras "não" e "saber", destacando-se os seguintes discursos: "não tenho informação sobre amamentação" e "não sei como fazer". Todos os profissionais da área da saúde - médicos, odontólogos, nutricionistas, e não só enfermeiros -, têm um papel fundamental para o sucesso do aleitamento materno. Em estudo, foi demonstrada a ação efetiva das visitas domiciliares dos profissionais de saúde para a melhora das relações interpessoais para a atenção e a orientação em saúde ${ }^{34}$. 
Tabela 3. Associação entre a intenção de aleitamento materno exclusivo por gestantes de alto risco e variáveis sociodemográficas. SP, 2018 ( $\mathrm{n}=1.118)$.

\begin{tabular}{|c|c|c|c|c|c|}
\hline & & \multicolumn{4}{|c|}{ Pretensão de amamentação exclusiva } \\
\hline \multicolumn{2}{|c|}{ Variáveis sociodemográficas } & $\begin{array}{c}\text { Não (69) } \\
\text { n (\%) } \\
\end{array}$ & $\begin{array}{c}\text { Sim (1.049) } \\
\mathbf{n}(\%)\end{array}$ & $\begin{array}{c}\text { Total (1.118) } \\
\mathbf{n}(\%)\end{array}$ & p valor ${ }^{a}$ \\
\hline \multirow{4}{*}{ Idade } & & & & & \\
\hline & $<20$ anos & $24(34,78)$ & $152(14,49)$ & $176(15,74)$ & $<0,0001$ \\
\hline & 20 a 34 anos & $37(53,63)$ & $649(61,87)$ & $686(61,36)$ & \\
\hline & $>34$ anos & $8(11,59)$ & $248(23,64)$ & $256(22,90)$ & \\
\hline \multirow[t]{4}{*}{ Renda Familiar } & Até 1500 reais & $47(68,12)$ & $483(46,05)$ & $530(47,40)$ & 0,0001 \\
\hline & De 1501 a 2500 reais & $12(17,39)$ & $357(34,03)$ & $369(33,01)$ & \\
\hline & Mais de 2500 & $4(5,80)$ & $177(16,87)$ & $181(16,19)$ & \\
\hline & $\begin{array}{l}\text { Não sabe/não } \\
\text { respondeu }\end{array}$ & $6(8,69)$ & $32(3,05)$ & $38(3,40)$ & \\
\hline \multirow[t]{4}{*}{ Escolaridade } & Até 7 anos & $22(31,88)$ & $162(15,44)$ & $184(16,46)$ & 0,0003 \\
\hline & De 8 a 10 anos & $26(37,68)$ & $319(30,41)$ & $345(30,86)$ & \\
\hline & 11 anos & $13(18,85)$ & $398(37,94)$ & $411(36,76)$ & \\
\hline & Mais de 11 anos & $8(11,59)$ & $170(16,21)$ & $178(15,92)$ & \\
\hline \multirow[t]{2}{*}{ Estado civil } & Solteira/Divorciada & $27(39,13)$ & $218(20,78)$ & $245(21,92)$ & 0,0006 \\
\hline & Casada/Amasiada & $42(60,87)$ & $831(79,22)$ & $873(78,08)$ & \\
\hline \multirow[t]{5}{*}{ Ocupação } & Autônoma & $4(5,80)$ & $80(7,63)$ & $84(7,52)$ & 0,0007 \\
\hline & Empregada & $17(24,64)$ & $431(41,09)$ & $448(40,07)$ & \\
\hline & Desempregada & $16(23,18)$ & $166(15,82)$ & $182(16,28)$ & \\
\hline & Do lar & $17(24,64)$ & $283(26,98)$ & $300(26,83)$ & \\
\hline & Estudante/pensionista & $15(21,74)$ & $89(8,48)$ & $104(9,30)$ & \\
\hline \multirow{3}{*}{$\begin{array}{l}\text { Semana } \\
\text { gestacional }\end{array}$} & Até 12 semanas & $5(7,24)$ & $119(11,34)$ & $124(11,09)$ & 0,4406 \\
\hline & De 13 a 26 semanas & $32(46,38)$ & $508(48,43)$ & $540(48,30)$ & \\
\hline & 27 semanas ou mais & $32(46,38)$ & $422(40,23)$ & $454(40,61)$ & \\
\hline
\end{tabular}

${ }^{a}$ Teste qui quadrado ou exato de Fisher, considerando $\mathrm{p}<0,05$.

A amamentação exclusiva demonstrou associação com as variáveis sociodemográficas, corroborando com estudos de gestantes de baixo risco $^{7,14,29}$. Em pesquisa, mães de idade avançada e que não tiveram orientação apresentaram de quatro a cinco vezes menos chances de amamentar seus bebês ${ }^{17}$. Tal informação diverge dos dados encontrados neste estudo, visto que gestantes mais novas relataram menor pretensão de aleitamento materno exclusivo. A falta de experiência em gestantes mais novas e o baixo nível de escolaridade, em conjunto com a falta de orientação sobre a amamentação, podem justificar este achado. Os motivos principais para a amamentação exclusiva, nesta pesquisa, foram relacionados à saúde, à imunidade e ao desenvolvimento do bebê, corroborando com outro estudo ${ }^{32}$.

$\mathrm{Na}$ literatura, diversas barreiras são apontadas para a amamentação. Dentre elas podem ser citados o tipo de parto, a falta de orientação, a baixa escolaridade, o medo da dor, as primigestas e o trabalho ${ }^{32,33}$. Algumas condições presentes na gestação de alto risco - como obesidade ou sobrepeso, depressão, uso de tabaco e adolescência - tam- bém apresentaram maiores chances de início tardio da amamentação por um período menor de tempo ${ }^{33,35-38}$. Por se tratarem de gestantes de alto risco neste estudo, a indicação de parto cesariana é mais prevalente, reforçando-se a necessidade da atenção especial para essas gestantes.

A duração pretendida para a amamentação demonstrou resultados semelhantes em outros estudos, evidenciando a necessidade de atenção especial, com relação ao aleitamento materno, para gestantes de baixa escolaridade e de baixa idade que não têm um companheiro ${ }^{19,27,29,33}$.

A associação entre o histórico de amamentação anterior e a duração em que as gestantes pretendiam amamentar seus bebês presentes neste estudo podem ser justificadas pelo sucesso da amamentação de outros filhos, visto que, em casos de insucesso, os medos e os anseios gerados pela experiência anterior poderiam interferir negativamente no aleitamento do próximo filho ${ }^{14}$.

A técnica metodológica de análise qualitativa empregada nesta pesquisa possibilitou aprofundar os conhecimentos sobre os motivos para a recusa do aleitamento materno exclusivo. 


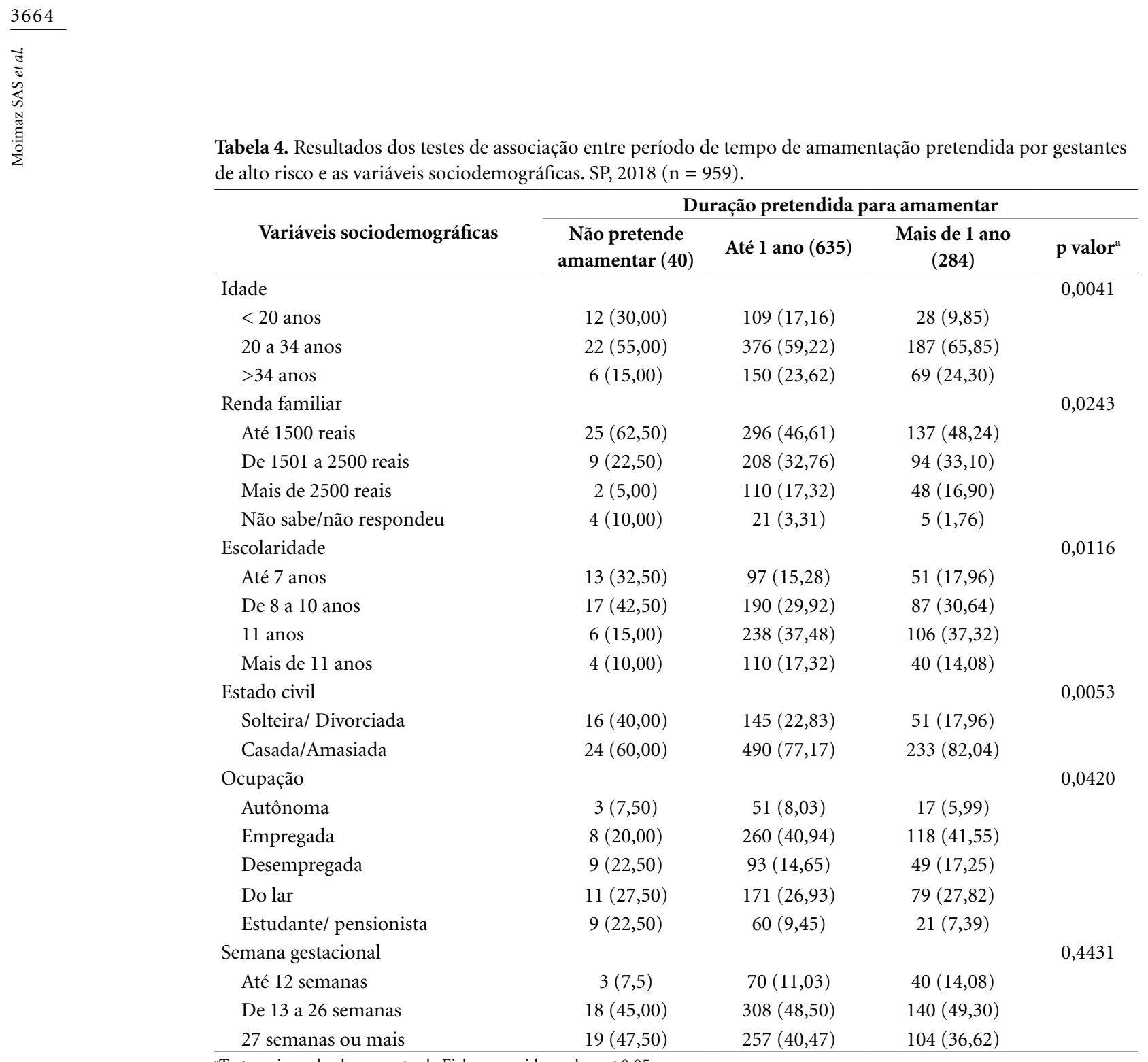

aTeste qui quadrado ou exato de Fisher, considerando $p<0,05$.

Observando a análise de similitude e a nuvem de palavras, a ocorrência de motivos como o HIV e o uso de remédio foram frequentes. No entanto, o que chama a atenção são as palavras "trabalho", "saber" e "água/mamadeira", que podem ser consideradas barreiras reversíveis para o aleitamento exclusivo. Algumas gestantes relataram o uso de drogas ilícitas e lícitas, mas nenhuma participante relatou esse motivo para o não aleitamento exclusivo. Trechos de discursos que evidenciam essas barreiras são "não faz mal dar água", "pretende alternar com leite em pó por causa da faculdade" e "porque vou trabalhar".

A introdução de outros líquidos além do leite materno nos primeiros quatro meses de vida do bebê não é recomendada, visto que pode interferir na absorção de nutrientes e diminuir a quantidade de leite materno ingerido, levando a um menor ganho de peso e ao aumento do risco de infecções gastrointestinais e respiratórias, além de alergias e diarreias ${ }^{16,19}$. Para algumas mães, a introdução de outros alimentos deveria ser feita desde o nascimento devido à crença de que a amamentação deixaria o bebê dependente do peito, dificultando o retorno da mãe ao trabalho, momento em que os bebês ficariam sob o cuidado de terceiros ${ }^{19-22}$.

O trabalho e a amamentação há muito tempo vêm sendo discutidos pelos órgãos mundiais de saúde, sendo tema central da Semana Mundial do Aleitamento ocorrida em 2015 $5^{5,21}$. Apesar de diversas leis e normativas visarem a garantir o aleitamento materno de mães que trabalham e a redução da inserção de bicos artificiais e outros leites na primeira infância, o trabalho ainda pode ser considerado como fator de risco para a interrup- 


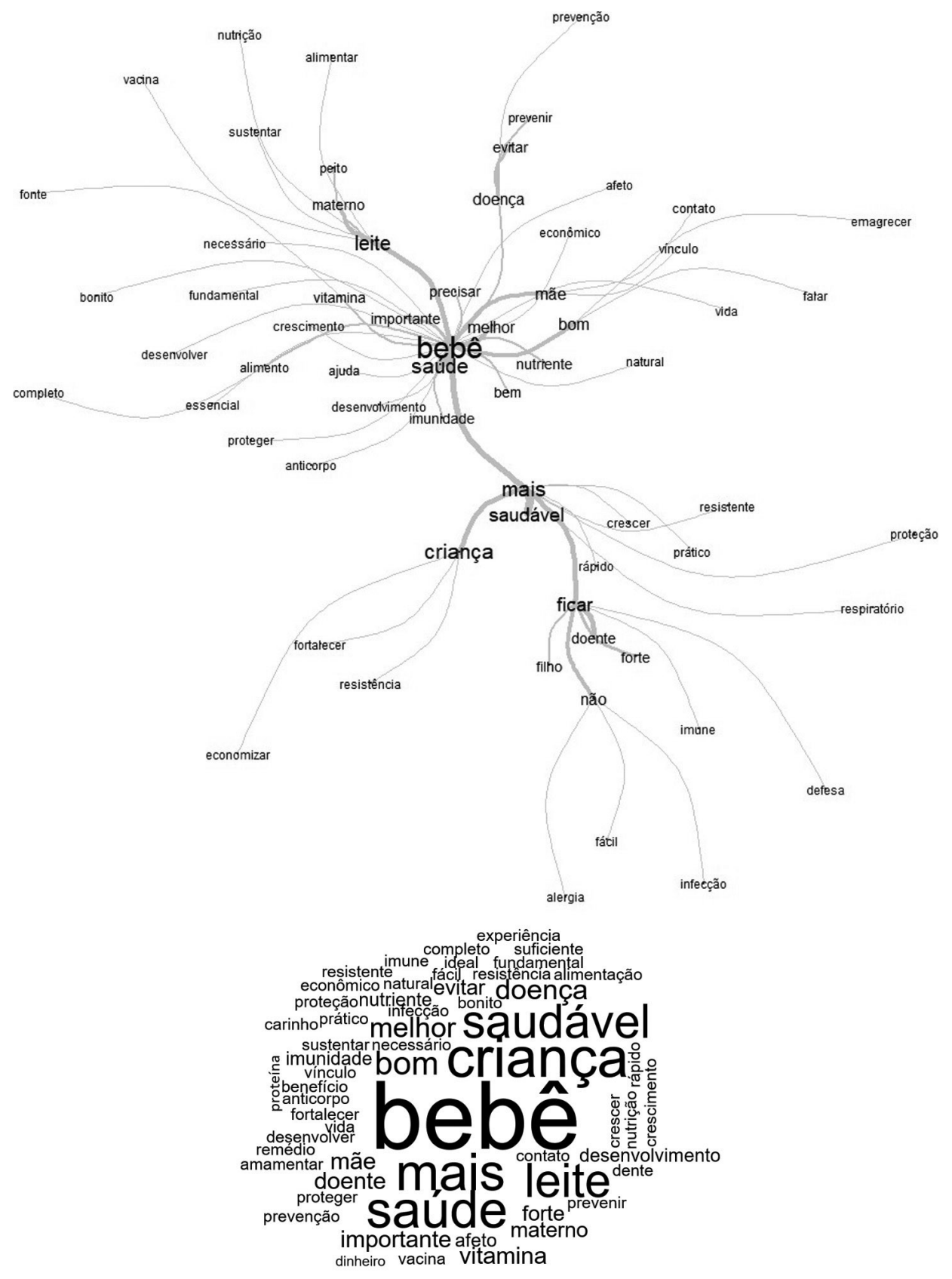

Figura 1. Análise de similitude e nuvem de palavras relacionadas aos motivos relatados para a amamentação exclusiva de gestantes de alto risco. SP, 2018.

ção da amamentação, como visto neste e em outros estudos, como os de Kalil e Aguiar ${ }^{21}$, Brasileiro et al. ${ }^{22}$ e Moimaz et al. ${ }^{33}$.

Neste estudo, a técnica empregada foi a pesquisa transversal, por meio de inquérito - o que pode ser considerada uma limitação, em função da emissão, por parte das gestantes, de respostas "esperadas". Para o acompanhamento dessas gestantes, novos estudos de seguimento, que visem a avaliar se a prática de aleitamento foi concretizada, 


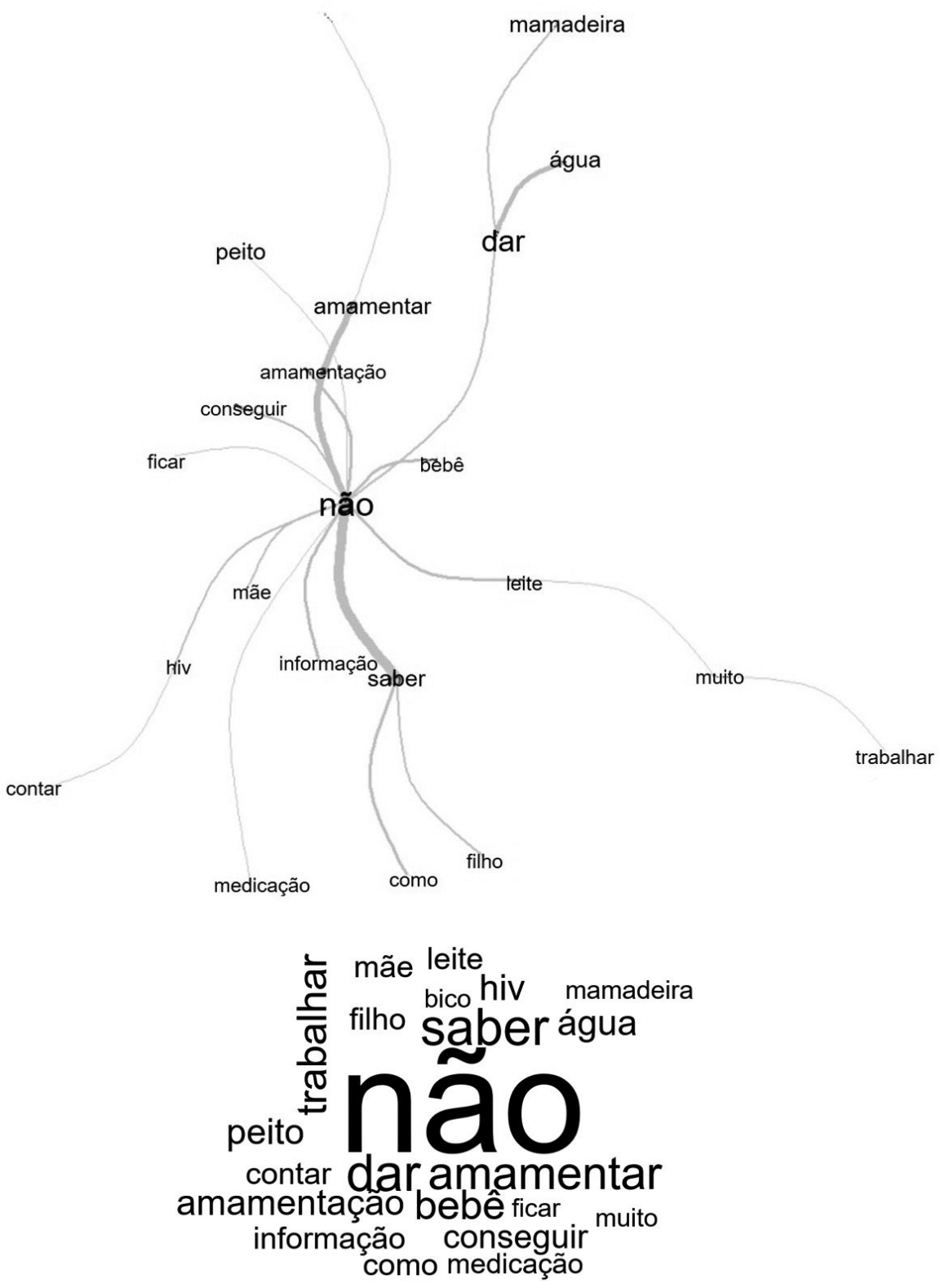

Figura 2. Análise de similitude e nuvem de palavras com principais motivos relatados para a não amamentação exclusiva de gestantes de alto risco. SP, 2018.

e outros estudos qualitativos com as mães que não amamentaram seus outros filhos devem ser realizados para complementar os achados deste estudo.

A orientação para gestantes sobre as contraindicações, sobre como manter o aleitamento após a volta ao trabalho e sobre os benefícios do aleitamento tanto para mãe como para o bebê por um período prolongado de tempo poderiam ter grande contribuição para o sucesso do aleitamento materno. 


\section{Conclusão}

Algumas gestantes desta pesquisa apresentaram condições para o alto risco gestacional que poderiam interferir ou contraindicar a amamentação.

Gestantes de alto risco apresentaram altas taxas de intenção de amamentação exclusiva, principalmente aquelas que possuíam escolaridade mais elevada, tiveram outros filhos amamentados e que contavam com apoio familiar. A maioria delas pretendiam amamentar exclusivamente até os seis meses de idade do bebê.

Os principais motivos relatados para a não intenção de amamentação exclusiva foram os seguintes: ser portadora do vírus do HIV, o uso de medicação, o trabalho e a falta de informações sobre a amamentação.

\section{Referências}

1. World Health Organization (WHO). Ten steps to successful breastfeeding (revised 2018) [Internet]. WHO [acessado 2018 Maio 9]. Disponível em: http://www. who.int/nutrition/bfhi/ten-steps/en/

2. Organização Panamericana de Saúde/Organização Mundial de Saúde do Brasil (OPAS/OMS) [Internet]. 2017 [acessado 2018 Jan 3]. Disponível em: http:// www.paho.org/bra/

3. Fundo das Nações Unidas para a Infância Brazil (UNICEF) [Internet]. [acessado 2018 Jan 3]. Disponível em: https://www.unicef.org/brazil/pt/

4. Brasil. Ministério da Saúde (MS). Saúde da criança: nutrição infantil aleitamento materno e alimentação complementar. Brasília: MS; 2009.

5. World Alliance for Breastfeeding Action. Protects, Promotes and Supports Breastfeeding Worldwide [Internet]. [acessado 2018 Maio 9]. Disponível em: http:// waba.org.my/

6. Brasil. Ministério da Saúde (MS). Iniciativa hospital amigo da criança: revista, atualizada e ampliada para o cuidado integrado. Fundo das nações unidas para a infância. Brasília: OMS; 2009.

7. Silva CM, Pellegrinelli ALR, Pereira SCL, Passos IR, Santos LC. Práticas educativas segundo os "Dez passos para o sucesso do aleitamento materno" em um Banco de Leite Humano. Cien Saúde Colet 2017; 22(5):1661-1671.

8. Brasil. Ministério da Saúde (MS). Portaria no 1.459 , de 24 de junho de 2011. Institui, no âmbito do Sistema Único de Saúde - SUS - a Rede Cegonha. Diário Oficial da União 2011; 24 jun.

\section{Colaboradores}

SAS Moimaz contribuiu com a elaboração e desenvolvimento da pesquisa, revisão bibliográfica, tabulação dos dados e correção final do texto. DT Ros contribuiu com o desenvolvimento da pesquisa, revisão bibliográfica, coleta e tabulação dos dados, redação do artigo. TA Saliba contribuiu com o desenvolvimento da pesquisa, revisão bibliográfica, correção final do texto. NA Saliba contribuiu com a elaboração e desenvolvimento da pesquisa, revisão bibliográfica, correção final do texto.

9. Oktaria V, Lee KJ, Bines JE, Watts E, Satria CD, Atthobari J, Nirwati H, Kirkwood D, Soenarto Y, Danchin MH. Nutritional status, exclusive breastfeeding and management of acute respiratory illness and diarrhea in the first 6 months of life in infants from two regions of Indonesia. BMC Pediatr 2017; 17(1):211.

10. Horta BL, Mola CL, Victora CG. Long-term consequences of breastfeeding on cholesterol, obesity, systolic blood pressure and type 2 diabetes: a systematic review and meta-analysis. Acta Paediatr 2015; 104(467):30-37.

11. Fonseca AL, Albernaz EP, Kaufmann CC, Neves IH, Figueiredo VL. Impacto do aleitamento materno no coeficiente de inteligência de crianças de oito anos de idade. Rev Soc Boliv Pediatr 2015; 54(1):41-49.

12. Dijigow FB, Paganoti CF, Costa RA, Francisco RPV, Zugaib M. Influência da amamentação nos resultados do teste oral de tolerância à glicose pós-parto de mulheres com diabetes mellitus gestacional. Rev Bras Ginecol Obstetr 2015; 37(12):565-570.

13. Moimaz SAS, Ros DT, Garbin CAS, Saliba NA. Aleitamento materno: benefícios à saúde e implicações na saúde bucal. Rev Paul Odontol 2014; 36(4):24-31.

14. Rocha NB, Garbin AJI, Garbin CAS, Moimaz SAS. $\mathrm{O}$ ato de amamentar: um estudo qualitativo. Physis 2010; 20(4):1293-1305.

15. Brasil. Ministério da Saúde (MS). Departamento de Ações Programáticas Estratégicas. Gestação de alto risco: manual técnico. Brasília: MS; 2012. 
16. Fonseca PCA, Carvalho CA, Ribeiro SAV, Nobre LN, Pessoa MC, Ribeiro AQ, Priore SE, Franceschini SCC. Determinantes da velocidade média de crescimento de crianças até seis meses de vida: um estudo de coorte. Cien Saúde Colet 2017; 22(8):2713-2726.

17. Caminha MFC, Cruz RSBLC, Acioly VMC, Nascimento RR, Azevedo PTÁCC, Lira PIC, Batista Filho M. Fatores de risco para a não amamentação: um estudo caso-controle. Rev Bras Saúde Materno Infant 2015; 15(2):193-199.

18. Mgongo M, Mosha MV, Uriyo JG, Msuya SE, Stray-Pedersen B. Prevalence and predictors of exclusive breastfeeding among women in Kilimanjaro region, Northern Tanzania: a population based cross-sectional study. Int Breastfeed J 2013; 8(1):12.

19. Flores TR, Nunes BP, Neves RG, Wendt AT, Costa CS, Wehrmeister FC, Bertoldi AD. Consumo de leite materno e fatores associados em crianças menores de dois anos: Pesquisa Nacional de Saúde, 2013. Cad Saúde Pública 2017; 33(11):e00068816.

20. Gomes JLGC, Rossetto EG, Souza SNDH, Scochi CGS. The prevalence of breastfeeding in prematures with very low birth weight - a systematic review. Online Braz J Nurs 2009; 8(2):1-8.

21. Kalil IR, Aguiar AC. Trabalho feminino, políticas familiares e discursos pró-aleitamento materno: avanços e desafios à equidade de gênero. Saúde Debate 2016; 40(110):208-223.

22. Brasileiro AA, Ambrosano GMB, Marba STM, Possobon RF. A amamentação entre filhos de mulheres trabalhadoras. Rev Saúde Pública 2012; 46(4):642-648.

23. Brandão $M N$, Souza ES, Brito RJV, Guimarães C MMS, Brandão MFR, Cavalcante MC. Challenges in preventing vertical HIV transmission in Petrolina, Pernambuco and Juazeiro, Bahia. Rev Bras Saúde Matern Infant 2016; 16(3):313-324.

24. Lima SDS, Silva LCS, Santos MV, Martins JP, Oliveira MC, Brasileiro ME. HIV na gestação: pré-natal, parto e puerpério. Cien Saúde 2017; 10(1):56.

25. Moreno C, Rea MF, Filipe EV. Mães HIV positivo e a não-amamentação. Rev Bras Saúde Matern Infant 2006; 6(2):199-208.

26. White AB, Mirjahangir JF, Horvath H, Anglemyer A, Read JS. Antiretroviral interventions for preventing breast milk transmission of HIV. Cochrane Database Syst Rev 2014; 10:CD011323.

27. Duijts L, Jaddoe VWV, Hofman A, Moll HA. Prolonged and Exclusive Breastfeeding Reduces the Risk of Infectious Diseases in Infancy. Pediatrics 2010; 126(1):e18-e25.

28. Contarato AAPF, Rocha EDM, Czarnobay SA, Mastroeni SSBS, Veugelers PJ, Mastroeni MF. Efeito independente do tipo de aleitamento no risco de excesso de peso e obesidade em crianças entre 12-24 meses de idade. Cad Saúde Pública 2016; 32(12):e00119015.

29. Rocci E, Fernandes RAQ, Rocci E, Fernandes RAQ. Breastfeeding difficulties and influence in the early weaning. Rev Bras Enferm 2014; 67(1):22-27.
30. Esteves TMB, Daumas RP, Oliveira MIC, Andrade CAF, Leite IC. Factors associated to breastfeeding in the first hour of life: systematic review. Rev Saúde Pública 2014; 48(4):697-708.

31. Moimaz SAS, Ramirez GTV, Saliba TA, Saliba O, Garbin CAS. Expectativa e prática materna do aleitamento exclusivo e a saúde bucal do bebê. Rev Cien Plur 2017; 3(2):30-41.

32. Vieira TO, Martins CC, Santana GS, Vieira GO, Silva LR. Intenção materna de amamentar: revisão sistemática. Cien Saúde Colet 2016; 21(12):3845-3858.

33. Moimaz SAS, Rocha NB, Garbin CAS, Rovida TA, Saliba NA. Factors affecting intention to breastfeed of a group of Brazilian childbearing women. Women Birth 2017; 30(2):e119-e124.

34. Marin MJS, Gomes R, Junior S, Carlos A, Nunes CRR, Cardoso CP, Otoni MP, Moravcik MY. O sentido da visita domiciliária realizada por estudantes de medicina e enfermagem: um estudo qualitativo com usuários de unidades de saúde da família. Cien Saúde Colet 2011; 16(11):4357-4365

35. Colombo L, Crippa B, Consonni D, Bettinelli M, Agosti V, Mangino G, Bezze EN, Mauri PA, Zanotta L, Roggero P, Plevani L, Bertoli D, Gianni ML, Mosca F. Breastfeeding Determinants in Healthy Term Newborns. Nutrients 2018; 10(1):48.

36. Thompson LA, Zhang S, Black E, Das R, Ryngaert $\mathrm{M}$, Sullivan S, Roth J. The association of maternal pre-pregnancy body mass index with breastfeeding initiation. Metern Child Health J 2013; 17(10):18421851.

37. Furman LM, Banks EC, North AB. Breastfeeding among high-risk inner-city African-American mothers: a riscky choice? Breastfeeding Med 2013; 8(1):5867.

38. Martin MA, Garcia G, Kaplan HS, Gurven MD. Conflict or congruence? Maternal and infant-centric factors associated with shorter exclusive breastfeeding durations among the Tsimane. Soc Sci Med 2016; 170:9-17.

Artigo apresentado em 03/07/2018

Aprovado em 18/01/2019

Versão final apresentada em 20/01/2019 